\title{
SYMPOSIUM ON BIORHEOLOGY
}

In connection with the Japan-United States Seminar on Hemorheology and Thrombosis, held at Kobe, a Symposium on Biorheology took place in Tokyo on 7 May, 1975 under the sponsorship of the Institute of Physical and Chemical Research. The topics included various fields of biorheology.

Professor A. L. Copley of the Polytechnic Institute of New York (joint authors: C. R. Huang and R. G. King) reported that the sedimentation rate of erythrocytes showed a maximum at a shear rate of $0 \cdot 1 \mathrm{sec}^{-1}$. Professor S. Chien of Columbia University reviewed the influence of cell concentration, cell aggregation and cell deformability on the non-Newtonian flow properties of blood. Professor Y. Nakamura of Chiba University (joint author: $\mathrm{H}$. Wayland of the California Institute of Technology) described an interesting technique for the determination of the diffusion coefficients of macromolecules in the cat mesentery. Professor Y. Hiramoto of the Tokyo Institute of Technology reviewed viscoelastic properties of protoplasm of the sea urchin egg. Professor K. Sakaoku of Tokyo University of Agriculture and Technology presented electron micrographic observations of the structure of fibrin fibrils during clotting of blood. Professor S. Taira and Dr. M. Sato of Kyoto University were scheduled to talk on mechanical behavior of blood vessel walls, but were unable to attend due to a rail strike. Dr. E. Fukada of the Institute of Physical and Chemical Research (joint author: H. Ueda) showed the temperature dependence of the peizoelectric effect observed in hydrated horn keratin. Dr. L. Breger of the Institute of Physical and Chemical Research and of the University of Illinois described a new type of piezoelectric polarization in bone and tendon observed under bending deformation.

The Seminar was informative and took place in a very friendly atmosphere. Despite the rail strike and the visit of Queen Elizabeth of Great Britain the meeting was exceptionally well attended.

\section{JAPAN-UNITED STATES SEMINAR ON HEMORHEOLOGY AND THROMBOSIS}

\author{
Report by EIICHI FUKADA, Wako, Saitama, Japan.
}

A Seminar entitled "Hemorheology and Thrombosis" was held at the Oriental Hotel, Kobe, Japan from 28 April to 2 May 1975. The meeting was organized by Professor S. Okamoto (Co-ordinator for Japan), Kobe University School of Medicine, and Professor A. L. Copley (United States Co-ordinator), Polytechnic Institute of New York, under the auspices of the Japan Society for the Promotion of Science and the National Science Foundation, U.S.A.

The communication of knowledge and ideas among scientists of different fields is important to advance the interdisciplinary science such as biorheology. One such opportunity was successfully provided by the Seminar. About eighty participants included, amongst others, physiologists, cell biologists, pathologists, physicians, surgeons, gynecologists, physicists and chemists. The participants from the United States were Professor Copley, accompanied by his daughter Una Dora, Professor Seaman, Professor Fung, Professor Chien, Professor Brinkhous, accompanied by his wife, Drs. Nicolae and Maia Simionescu, and Professor Huang. The Seminar was also stimulated by the presence of Professor Birger Blombäck from Sweden and Professor Hartert from Germany accompanied by his wife.

The Seminar was divided into nine sessions and the topics covered wide areas of hemorheology and thrombosis: rheological properties of blood, dynamic measurements of blood clotting, physicochemical properties of erythrocytes, platelet interactions, flow of blood related to thrombosis, interaction of cells with vessel walls, transendothelial diffusion of macromolecules, thrombin inhibitors, activators of prothrombin and of plasminogen, structure and function of fibrinogen, thrombus formation, the genesis of thrombosis, fibrinolysis, and clinical problems.

As a one-day excursion during the Seminar, a visit to Naruto in Awaji Island was arranged. A vortex of sea currents gave a real demonstration of tidal rheology and "rock thrombosis". The enthusiastic efforts of Professor S. Okamoto, Dr. O. Matsuo and many other members of Kobe University, in organizing this international Seminar and in making it so enjoyable, are greatly appreciated.

The Proceedings of the Seminar will soon be published as Supplement II of Thrombosis Research, as well as in book form, edited by Professors A. L. Copley and S. Okamoto with the help of Dr. O. Matsuo.

The programme of the Seminar was as follows:

C. R. Huang (New Jersey Institute of Technology), Thixotropic Parameters of Whole Human Blood.

E. Fukada and M. Kaibara (The Institute of Physical and Chemical Research), Dynamic Measurements of Blood Coagulation.

T. Matsuda and M. Murakami* (Tokyo Metropolitan Institute of Gerontology and Tokyo Metropolitan Yoikuin Geriatrics Hospital*), Relationship Between Fibrinogen and Blood Viscosity.

H. H. Hartert (Saar-Universität and Städt. Krankenhaus Kaiserslautern), New Aspects of Blood Clotting and Thrombosis. Investigations with the Method of Rheosimulation.

S. Okamoto, A. Hijikata, K. Ikezawa, R. Kikumoto, ${ }^{*}$ S. Tonomura* and S. Tamao* (Kobe University School of Medicine 
and Mitsubishi Chemical Co.*), A New Series of Synthetic Thrombin-Inhibitors Assuming Extremely Potent and Selective Action.

A. Hijikata, S. Okamoto, E. Mori, R. Kikumoto, ${ }^{*}$ S. Tonomura, ${ }^{*}$ Y. Tamao, ${ }^{*}$ and H. Hara* (Kobe University School of Medicine and Mitusbishi Chemical Ind.*), In Vitro and In Vivo Action of a New Series of Synthetic Thrombin-Inhibitors.

M. Kazama and T. Abe (Teikyo University School of Medicine), Metabolic Aspect of Fibrinogen and F. XIII in the Process of Disseminated Intravascular Coagulation.

S. Iwanaga, T. Morita and T. Suzuki (Institute for Protein Research, Osaka University), Activation of Bovine Prothrombin by Echis carinatus Venom Activator.

B. Blombäck (The New York Blood Center and Karolinska Institute), Structure and Function of Fibrinogen.

S. Izaki and H. Kitaguchi (Kobe University School of Medicine), Releasing Mechanism of Plasminogen Activator from the Vascular Wall, with Reference to Vasoconstriction.

H. Soma (Tokyo Medical College), The Coagulation System During Placental Separation.

M. Maki, M. Nagayama, T. Oda and S. Shinagawa (Hirosaki University School of Medicine), Anticoagulant Release During Shock.

S. Tojo, and H. Fukunishi (Kobe University School of Medicine), In Vitro Perfusion of Human Reproductive Organs.

M. Kaibara and E. Fukada (The Institute of Physical and Chemical Research), Dynamic Viscoelasticity of Fibrin Gels; Dependence on Ionic Strength.

N. Ohshima, S. Kanasaki and M. Hori (University of Tsukuba and Tokyo Women's Medical College), Hemorheology in Hyper- and Hypo-Viscosity Syndrome.

Y. Isogai (Jikei University School of Medicine), Hemorheological Studies on the Pathogenesis of Diabetic Microangiopathy.

S. Chien (Columbia University College of Physicians and Surgeons), Electrochemical Interactions between Erythrocyte Surfaces.

T. Tsukada (Toranomon General Hospital), Platelet Retention in Glass Bead Columns: Its Mechanism and the Effect of Plasma Proteins.

H. Yamazaki, T. Sano, T. Asano* and H. Hidaka* (Tokyo Medical and Dental University and Aichi Prefecture Colony*), Hyperaggregability of Plateletes in Thromboembolic Disorders.

T. Maekawa, N. Kobayashi and H. Arai (Gunma University School of Medicine), Role of Blood Platelet in the Formation of Thrombus.

K. Yasunaga (Kyoto University), Integrated Analysis of Hemorrhagic and Thrombotic Tendency.

G. V. F. Seaman (University of Oregon Health Sciences Center), Electrochemical Features of Platelet Interactions.

M. Simionescu, N. Simionescu, and G. E. Palade (Yale University School of Medicine), Characteristic Endothelial Junctions in Different Segments of the Vascular System.

T. Murata (Tokyo Metropolitan University), A Theoretical Analysis of Material Transport across a Capillary Wall.

A. Sakanishi, S. Mitaku and Y. Takano (University of Tokyo), Linear Viscoelasticity in Suspensions of Spherical Shell Structures and Erythrocytes.

K. Taniguchi (Tokyo Med. \& Dent. University), Studies on the Orifice Flow Through the Stenosis of Vessels.

N. Simionescu, M. Simionescu and G. E. Palade (Yale University School of Medicine), Transendothelial Exchange of Macromolecules.

A. Kuramoto, T. Taketomi and J. Nakagawa (Research Institute for Nuclear Medicine and Biology, Hiroshima University), Metabolic and Kinetic Study of Thrombogenesis; Regulatory Mechanism in Early Step of Platelet-plug Formation.

G. Ooneda, Y. Yoshida, K. Suzuki and H. Shinkai (Gunma University, School of Medicine), Plasmatic Arterionecrosis and its Thrombotic Occlusion.

K. Tanaka and A. Sumiyoshi (Kyushu University), Intimal Injury and Thrombosis.

K. M. Brinkhous (University of North Carolina), Possible Role of von Willebrand Factor in White Thrombus Formation.

O. Matsuo and Y. Rokushima (Kobe University School of Medicine), Some Aspects of Dose-Response of UK Therapy.

Y. Abiko and T. Kumada (Daiichi Seiyaku Co.), Enhancement of Fibrinolytic and Thrombolytic Potential in the Rat by an Anabolic Steroid, Furazabol.

K. Yamada, A. Shirahata and T. Meguro (Keio University and Ogikubo Hospital), The Effects of an Alpha Blocking Agent on the Rds with Dic in the Newborn Infants and Experimental Animals.

T. Abe and M. Kazama (Teikyo University School of Medicine), Defibrination Syndrome as Drastic Deviation of Coagulation/Fibrinolysis Balance and Its Therapeutic Management.

A. L. Copley (Polytechnic Institute of New York), Polymolecular Layers of Fibrinogen Systems and the Genesis of Thrombosis.

A. Kikuchi (Tokyo Hitachi Hospital), Rheological Observation on the Development of Mechanically Induced Thrombus.

T. Azuma and T. Fukushima (Shinshu University Medical School), Disturbance of Blood Flow as a Factor of Thrombus Formation.

Y. Matunobu (Keio University) A Theoretical Estimation of the Plasma Layer in Arteriolar Blood Flow.

Y. B. Fung (University of California, San Diego), Interaction of Blood Cells with Vessel Walls in Microcirculation.

S. Oka (Kyorin University), A Theoretical Approach to the Effect of Shear Stress on the Development of Atheroma. 